\title{
Tyrosine Nitration within the Proline-Rich Region of Tau in Alzheimer's Disease
}

\author{
Juan F. Reyes, ${ }^{*}$ Yifan Fu, ${ }^{*}$ Laurel Vana, ${ }^{*}$ \\ Nicholas M. Kanaan, ${ }^{\dagger}$ and Lester I. Binder ${ }^{* \neq}$ \\ From the Department of Cell and Molecular Biology, ${ }^{*}$ and the \\ Cognitive Neurology and Alzheimer's Disease Center, Feinberg \\ School of Medicine, Northwestern University, Chicago, Illinois; \\ and the Division of Translational Science and Molecular \\ Medicine, ${ }^{\dagger}$ College of Human Medicine, Michigan State \\ University, Grand Rapids, Michigan
}

A substantial body of evidence suggests that nitrative injury contributes to neurodegeneration in Alzheimer's disease (AD) and other neurodegenerative disorders. Previously, we showed in vitro that within the tau protein the $\mathrm{N}$-terminal tyrosine residues (Y18 and Y29) are more susceptible to nitrative modifications than other tyrosine sites (Y197 and Y394). Using sitespecific antibodies to nitrated tau at Y18 and Y29, we identified tau nitrated in both glial (Y18) and neuronal (Y29) tau pathologies. In this study, we report the characterization of two novel monoclonal antibodies, Tau-nY197 and Tau-nY394, recognizing tau nitrated at Y197 and Y394, respectively. By Western blot analysis, Tau-nY197 labeled soluble tau and insoluble paired helical filament proteins (PHF-tau) nitrated at Y197 from control and AD brain samples. TaunY394 failed to label soluble tau isolated from control or severe AD samples, but labeled insoluble PHF-tau to a limited extent. Immunohistochemical analysis using Tau-nY197 revealed the hallmark tau pathology associated with AD; Tau-nY394 did not detect any pathological lesions characteristic of the disorder. These data suggest that a subset of the hallmark pathological inclusions of $\mathrm{AD}$ contain tau nitrated at Y197. However, nitration at Y197 was also identified in soluble tau from all control samples, including those at Braak stage 0 , suggesting that nitration at this site in the proline-rich region of tau may have normal biological functions in the human brain. (Am J Pathol 2011, 178:2275-2285; DOI: 10.1016/j.ajpath.2011.01.030)

Alzheimer's disease (AD) is a progressive neurodegenerative disorder and the most common form of dementia in the elderly. Pathologically, this disease is character- ized by the formation of neurofibrillary tangles and amyloid plaques, comprised primarily of modified tau and amyloid- $\beta$ proteins, respectively. ${ }^{1,2}$ Tau is a microtubule associated protein (MAP) encoded on chromosome 17; alternative splicing of the tau message leads to six canonical isoforms in the central nervous system containing zero, one, or two $\mathrm{N}$-terminal inserts and either three or four microtubule binding repeats. ${ }^{3,4}$ In $\mathrm{AD}$, all tau isoforms comprise neuropil threads, neuritic plaques, and neurofibrillary tangles (NFTs), ${ }^{5}$ which are the hallmark pathological tau inclusions that correlate with neuronal dysfunction and cognitive decline during the disease process. ${ }^{6,7}$

The aggregation potential of tau into fibrillar structures is influenced by various modifications within the molecule. For instance, site-specific phosphorylation, proteolytic cleavage, or specific conformations can enhance tau aggregation and toxicity. ${ }^{8-11}$ Tyrosine nitration in proteins has recently received considerable attention, after this posttranslational modification was identified within tau in $A D$ and in proteins involved in other neurodegenerative disorders $^{12,13}$; however, the mechanism for tyrosine nitration and its role in protein aggregation in vivo remain highly controversial. ${ }^{14-19}$ Several studies suggest that tyrosine nitration is a random pathological event that occurs as a result of free radical generation during the neurodegenerative processes in disease. ${ }^{14,20}$ Under these conditions, reactive nitrogen and oxygen species are thought to be generated to form peroxynitrite $\left(\mathrm{ONOO}^{-}\right)$, a highly reactive compound capable of inducing nitrative and oxidative protein modifications. ${ }^{21}$ Indeed, the reaction of $\mathrm{ONOO}^{-}$with proteins in vitro can lead to the formation of 3-nitrotyrosine (3-NT) or higher-order aggregates by the oxidative addition of two tyrosyl radicals to form 3,3'dityrosine $\left(3,3^{\prime}\right.$-DT) cross-linked proteins. ${ }^{22-24}$

Alternatively, other studies suggest that protein tyrosine nitration might have a normal biological function, given that a number of cytoskeletal proteins are nitrated in different

Supported by awards from the NIH (AG014449 and AG032091 to L.I.B.; T32 AG000260 to J.F.R.).

Accepted for publication January 12, 2011.

Supplemental material for this article can be found at http://ajp. amjpathol.org or at doi: 10.1016/j.ajpath.2011.01.030.

Address reprint requests to Juan F. Reyes, M.S., Tarry Bldg. 8-754, 300 E Superior, Chicago, IL 60611. E-mail: juan-reyes@northwestern.edu. 
cell types under normal conditions. ${ }^{25-27}$ For instance, in the chicken embryo, actin and $\alpha$-tubulin are differentially nitrated during normal development. ${ }^{28}$ Tau nitrated at tyrosine 197 has been identified in wild-type 6-week-old mice using mass-spectrometry analysis. ${ }^{27}$ Based on these and other studies, the potential existence of an enzymatic pathway for tyrosine nitration has been proposed. 26,29

Tau contains five endogenous tyrosine residues, located at positions 18, 29, 197, 310, and 394 (numbers correspond to the longest tau isoform, which contains 441 amino acids). In vitro, $\mathrm{ONOO}^{-}$-mediated tyrosine nitration occurs selectively at tyrosine residues located within the most $\mathrm{N}$-terminal region of the tau molecule ( $\mathrm{Y} 18$, Y29) and to a lesser extent at Y197 and Y394. Nitration at Y310 was seldom observed, probably because of its location within the hydrophobic microtubule binding repeat of tau. $^{24,30 ~ I n ~ s i t u, ~ Y 18 ~ a n d ~ Y 29 ~ w e r e ~ s h o w n ~ t o ~ b e ~ n i t r a t e d ~ i n ~}$ AD using Tau-nY18 and Tau-nY29 antibodies, and nitrated proteins were selectively localized to the glial $(\mathrm{Y} 18)$ and neuronal (Y29) tau pathologies. ${ }^{31,32}$

To investigate whether Y197 and Y394 are nitrated in human brain, we generated two novel monoclonal antibodies, Tau-nY197 and Tau-nY394, that are specific to tau nitrated at Y197 and Y394, respectively. By Western blot analysis, Tau-nY197 identified soluble tau from controls (Braak stages 0 to III) and from severe AD cases (Braak stages $\mathrm{V}$ to $\mathrm{VI}$ ). However, Tau-nY394 failed to label soluble tau in any of the samples analyzed. Both Tau-nY197 and Tau-nY394 labeled insoluble paired helical filament proteins (PHF-tau) isolated from severe $A D$ cases (Braak stages $\mathrm{V}$ to $\mathrm{VI}$ ), although the Tau-nY394 signal was very weak.

Immunohistochemical analysis of postmortem brain tissue using Tau-nY197 demonstrated that the hallmark tau pathology of $A D$ contained tau nitrated at $Y 197$; this antibody also colocalized to a limited extent with tau markers for the early stages of tangle formation. However, Tau-nY394 failed to detect any pathological tau inclusions. Collectively, our data suggest that nitration at Y197 within tau may have normal biological function, because it occurs in seemingly normal unaffected brain tissue and persists throughout the progression of the disease process in both soluble and aggregated tau species.

\section{Materials and Methods}

\section{Antibody Production}

Mouse monoclonal antibodies were raised against synthetic peptides $\left({ }^{191}\right.$ SGDRSGY ${ }^{\mathrm{NO}}$ SSPGS ${ }^{202}$ or ${ }^{387}$ GAEIVYNO2 KSPVVC ${ }^{400}$ ) harboring a nitro group $\left(-\mathrm{NO}_{2}\right)$ at tyrosine 197 or 394 (Cell Essentials, Boston, MA) and were prepared as described previously. ${ }^{31,32}$ Briefly, C57/B6 female tau knockout mice (Jackson Laboratories, Bar Harbor, ME) were immunized subcutaneously with peptides every 21 days until good titer was achieved; splenocytes were then removed and fused to SP2/o myeloma cells as described previously. ${ }^{33}$ Positive clones that reacted specifically with tau nitrated at Y197 or at Y394 by enzymelinked immunosorbent assay (ELISA) and Western blot analyses were selected and then were subcloned four times at limiting dilutions before antibody purification. Along with generating the Y197 nitration-specific antibody, a clone was also isolated that produced a nitrationindependent antibody that labeled total tau (regardless of the nitration state).

\section{Recombinant Tau Proteins}

Mutant and wild-type proteins were expressed in T7competent Escherichia coli cells (New England Biolabs, Ipswich, MA), using the pT7C-ht40 plasmid described previously. ${ }^{4}$ This vector contains cDNA encoding fulllength human tau (ht40; 441 amino acids) with an N-terminal polyhistidine tag. Five mutant proteins were generated, each containing only one of the five native tyrosines in tau $(18,29,197,310$, or 394). For each mutant, four of the five tyrosine residues were mutated to phenylalanine $(F)$, or in some instances to glutamic acid $(E)$, using site-directed mutagenesis (Stratagene, La Jolla, CA); the integrity of all cDNA constructs was verified by DNA sequencing.

\section{Tyrosine Nitration}

Mutant and wild-type proteins were treated with peroxynitrite to nitrate the available tyrosine residue or residues as described previously. ${ }^{31,32}$ Briefly, purified proteins were buffer-exchanged into nitration buffer $(100 \mathrm{mmol} / \mathrm{L}$ potassium phosphate, $25 \mathrm{mmol} / \mathrm{L}$ sodium bicarbonate, and 0.1 $\mathrm{mmol} / \mathrm{L}$ diethylenetriaminepentaacetic acid), ${ }^{20}$ then treated with 100-fold molar excess of peroxynitrite (Cayman $\mathrm{Co}$, Arbor, $\mathrm{Ml}$ ) at room temperature with constant stirring and stored at $-80^{\circ} \mathrm{C}$ until further use. Peroxynitrite treatment resulted in proteins nitrated at all tyrosines in wild-type tau (nht40) and in mutant proteins at $\mathrm{Y} 18$ (nY18), Y29 (nY29), Y197 (nY197), and Y394 (nY394).

\section{Tyrosine Phosphorylation}

Mutant proteins containing a single tyrosine residue were incubated with $\mathrm{Abl}$, a tyrosine kinase capable of phosphorylating tyrosine residues in vitro, according to the manufacturer's instructions (New England Biolabs). Addition of phosphate groups to proteins was confirmed using a generic phospho-tyrosine antibody (P-Tyr-100), as specified by the manufacturer (Cell Signaling Technologies, Danvers, MA).

\section{ELISA}

The specificity of monoclonal antibodies was tested using ELISA by attaching various nitrated and non-nitrated proteins diluted in borate saline buffer $(100 \mathrm{mmol} / \mathrm{L}$ boric acid, $25 \mathrm{mmol} / \mathrm{L}$ sodium borate, $75 \mathrm{mmol} / \mathrm{L} \mathrm{NaCl}, \mathrm{pH} 7.4)$ to 96-well microtiter plates (100 ng/well) (Corning Life Sciences, Lowell, MA). After blocking for 1 hour in 5\% nonfat dry milk (diluted in borate saline buffer), plates were incubated overnight at $4^{\circ} \mathrm{C}$ with primary antibodies. Plates were then washed and incubated with a peroxi- 
Table 1. Cases Used for the Enrichment of Soluble and Insoluble PHF-Tau

\begin{tabular}{cccc}
\hline Case identifier & Sex & $\begin{array}{c}\text { Age } \\
\text { (years) }\end{array}$ & Braak stage \\
\hline 1 & $\mathrm{M}$ & 61 & 0 \\
2 & $\mathrm{M}$ & 62 & 0 \\
3 & $\mathrm{M}$ & 56 & $\mathrm{I}$ \\
4 & $\mathrm{M}$ & 61 & $\mathrm{I}$ \\
5 & $\mathrm{M}$ & 67 & $\mathrm{I}$ \\
6 & $\mathrm{M}$ & 66 & $\mathrm{I}$ \\
7 & $\mathrm{M}$ & 81 & $\mathrm{I}$ \\
8 & $\mathrm{M}$ & 77 & $\mathrm{I}$ \\
9 & $\mathrm{M}$ & 88 & $\mathrm{II}$ \\
10 & $\mathrm{~F}$ & 84 & $\mathrm{II}$ \\
11 & $\mathrm{M}$ & 77 & $\mathrm{~V}$ \\
12 & $\mathrm{~F}$ & 84 & $\mathrm{~V}$ \\
13 & $\mathrm{~F}$ & 87 & $\mathrm{~V}$ \\
14 & $\mathrm{M}$ & 81 & $\mathrm{VI}$ \\
15 & $\mathrm{~F}$ & 89 & $\mathrm{VI}$ \\
\hline
\end{tabular}

The cases of 10 nondemented, age-matched control subjects (Braak stages $0-$ III) and 5 severe Alzheimer's disease patients (Braak stages $\mathrm{V}-\mathrm{VI}$ ) were used to determine the presence of tau nitrated at $\mathrm{Y} 197$ and Y394 using biochemically enriched soluble and insoluble PHF-tau preparations

$M$, male; $F$, female

dase-conjugated goat anti-mouse IgG $\mathrm{H}+\mathrm{L}$ secondary antibody (Vector Laboratories, Burlingame, CA) for 1 hour at room temperature before washing and subsequent reaction with a $3^{\prime}, 3^{\prime}, 5,5^{\prime}$-tetramethylbenzidine substrate for 10 minutes at room temperature (Sigma-Aldrich, St. Louis, MO). The reaction was stopped using 3\% $\mathrm{H}_{2} \mathrm{SO}_{4}$ solution, and the absorbance was determined at $450 \mathrm{~nm}$ in an Ultra microplate reader (Bio-Tek Instruments, Winooski, VT).

\section{Tau Enrichment from Human Brain}

Soluble tau was enriched from the frontal cortex of normal control and severe AD samples (Table 1) as described previously. ${ }^{5,31,34}$ Briefly, gray matter from the frontal cortex was homogenized in a buffer containing $100 \mathrm{mmol} / \mathrm{L}$ 2-( $\mathrm{N}$-morpholino)ethanesulfonic acid (MES), $0.5 \mathrm{mmol} / \mathrm{L}$ $\mathrm{MgCl}_{2}, 1 \mathrm{mmol} / \mathrm{L}$ EGTA, $1 \mathrm{~mol} / \mathrm{L} \mathrm{NaCl}, 50 \mathrm{mmol} / \mathrm{L} \mathrm{D}-\mathrm{N}$ acetylglucosamine, $50 \mathrm{mmol} / \mathrm{L}$ imidazole, $25 \mathrm{mmol} / \mathrm{L}$ $\beta$-glycerophosphate, $20 \mathrm{mmol} / \mathrm{L} \mathrm{NaF}, 10 \mathrm{mmol} / \mathrm{L}$ sodium pyrophosphate, $0.5 \mathrm{mmol} / \mathrm{L}$ phenylmethanesulfonyl fluoride (PMSF), $\mathrm{pH}$ 6.5, and was centrifuged at 27,000 $\times 9$ for 30 minutes to remove nuclear and membrane-bound material. These soluble whole-brain homogenates were adjusted to $2 \% \beta$-mercaptoethanol $(\mathrm{v} / \mathrm{v})$ and then were placed in a boiling water bath for 10 minutes. After heat treatment, the samples were cooled over ice and centrifuged as above; the pellets were discarded. The supernatant containing soluble proteins was precipitated with $50 \%$ ammonium sulfate. After centrifugation at 27,000 $\times$ $g$ for 30 minutes, the pellets were resuspended and dialyzed in homogenization buffer overnight. The resulting extracts were adjusted to $2.5 \%$ perchloric acid before centrifugation as above. The resultant supernatants containing enriched soluble tau proteins were dialyzed in homogenization buffer at $4^{\circ} \mathrm{C}$ overnight before concentration in Amicon YM-10 spin columns (Millipore, Billerica, MA).
To enrich for insoluble PHF-tau fractions (Table 1), samples from five pathologically severe $A D$ patients (Braak stages $\mathrm{V}$ to $\mathrm{VI}$ ) were obtained from Rush University Medical Center and the Cognitive Neurology and Alzheimer's Disease Center at Northwestern University and were prepared as described elsewhere. ${ }^{31,32}$ Gray matter from frontal cortex was homogenized and centrifuged at 27,000 $\times g$ for 30 minutes to remove nuclear and membrane-bound material. The supernatants were centrifuged at $95,000 \times g$ for 2 hours, resulting in a pellet enriched in insoluble PHF-tau. The pellets were then resuspended into extraction buffer $(4 \mathrm{~mol} / \mathrm{L}$ guanidine- $\mathrm{HCl}$, $10 \mathrm{mmol} / \mathrm{L} \mathrm{Tris}-\mathrm{HCl}, 10 \mathrm{mmol} / \mathrm{L}$ dithiothreitol, $50 \mathrm{mmol} / \mathrm{L}$ D- $N$-acetylglucosamine, $50 \mathrm{mmol} / \mathrm{L}$ imidazole, $25 \mathrm{mmol} / \mathrm{L}$ $\beta$-glycerophosphate, $20 \mathrm{mmol} / \mathrm{L} \mathrm{NaF}, 10 \mathrm{mmol} / \mathrm{L}$ sodium pyrophosphate, and $0.5 \mathrm{mmol} / \mathrm{L} \mathrm{PMSF}, \mathrm{pH} 7.4$ ) and allowed to incubate for 2 hours at room temperature. Samples were then centrifuged for 1 hour at 95,000 $\times g$, and supernatant was dialyzed overnight at $4^{\circ} \mathrm{C}$ against BTP buffer $(20 \mathrm{mmol} / \mathrm{L}$ bis-Tris propanone, $1 \mathrm{mmol} / \mathrm{L}$ dithiothreitol, $\mathrm{pH}$ 7.0). The samples were then boiled for 10 minutes, cooled over ice, and centrifuged for 1 hour as above. Supernatants containing PHF-tau were then concentrated and stored at $-80^{\circ} \mathrm{C}$ until further use.

\section{Nitro Group Reduction and Antibody Absorption}

To reduce the nitro groups $\left(-\mathrm{NO}_{2}\right)$ into nonreactive amines $\left(\mathrm{NH}_{2}\right)$, nitrocellulose membranes containing nY197 or nY394 proteins were treated with dithionite (sodium hydrosulfite) under argon gas as described previously. ${ }^{31,35}$ Next, membranes were washed and blocked in 5\% nonfat dry milk in Tris buffer saline, then blotted with Tau-nY197, Tau-nY394, or 3-NT antibodies. Antibody absorption analysis was performed using $5 \mu \mathrm{g}$ of untreated or singly nitrated proteins at Y197 or Y394 overnight at $4^{\circ} \mathrm{C}$. Preabsorbed antibodies were then used to blot membranes containing singly nitrated proteins.

\section{Western Blot Analysis}

Samples were separated using 10\% SDS polyacrylamide gel electrophoresis and were transferred to nitrocellulose membranes for immunoblot analysis (Trans-Blot; Bio-Rad Laboratories, Hercules, CA). In some instances, membranes containing human tau samples were treated with calf intestinal phosphatase (CIP; $10 \mathrm{U} / \mathrm{mL}$ in $100 \mathrm{mmol} / \mathrm{L}$ Tris-buffered saline) at $4^{\circ} \mathrm{C}$ overnight to remove phosphate groups (New England Biolabs). Membranes were then blocked with 5\% nonfat dry milk in Tris-buffered saline and incubated with primary antibodies at $4^{\circ} \mathrm{C}$ overnight. After a rinsing, membranes were incubated in peroxidase-conjugated goat anti-mouse IgG $\mathrm{H}+\mathrm{L}$ secondary antibodies (Vector Laboratories) for 1 hour at room temperature, then washed and incubated with enzymatic chemiluminescence substrate (Pierce ECL; Thermo Fisher Scientific; Rockford, IL). Signal was visualized on HyBlot CL autoradiography film (VWR International, Batavia, IL). 


\section{Immunohistochemistry}

Tissue from the inferior temporal gyrus, hippocampus, and entorhinal cortex of eight patients with severe $A D$ (Braak stages $\mathrm{V}$ to $\mathrm{VI}$ ) and five age-matched control subjects (Braak stages I to III), fixed in $4 \%$ paraformaldehyde, was obtained as free-floating $40-\mu \mathrm{m}$-thick sections from the Cognitive Neurology and Alzheimer's Disease Center at Northwestern University. Tissue sections were incubated with CIP to remove phosphate groups as described above and were processed for $\mathrm{IHC}$ as described previously. ${ }^{31,32,36}$ Sections were then incubated with Tau-nY197 (40 ng/mL), Tau-Y197 (1 $\mu \mathrm{g} / \mathrm{mL})$, or TaunY394 (cell culture medium) at $4^{\circ} \mathrm{C}$ overnight. After a rinsing in PBS ( $\mathrm{pH} 7.4)$, sections were incubated with biotinylated goat-anti-mouse antibodies ( $\mu$-chain or $\gamma$-chain specific) according to the manufacturer's instructions (Jackson ImmunoResearch, West Grove, PA), followed by a 1-hour incubation in avidin-biotin complex solution (Vector Laboratories). Sections were then developed with metal-enhanced 3,3'-diaminobenzine (Pierce; Thermo Fisher Scientific) and mounted onto glass slides, air-dried overnight, dehydrated through graded alcohols, cleared with xylenes, and coverslipped with Permaslip (Alban Scientific, St. Louis, MO). The primary antibody dilutions used were predetermined by testing serial dilutions on adjacent tissue sections to assure the use of subsaturating concentrations.

\section{Immunofluorescence}

Double-label immunofluorescence was performed as described previously. ${ }^{31,32}$ CIP-treated sections were incubated with Tau-nY197 (20 ng/mL) and either Tau-Y197 (1 $\mu \mathrm{g} / \mathrm{mL})$, Alz-50 (0.2 $\mu \mathrm{g} / \mathrm{mL})$, Tau-MN423 (1 $\mu \mathrm{g} / \mathrm{mL})$, or Tau-C3 $(0.4 \mu \mathrm{g} / \mathrm{mL})$ antibodies overnight at $4^{\circ} \mathrm{C}$. Sections were then incubated with either fluorescein isothiocyanate-conjugated goat anti-mouse $\gamma$ chain-specific or Texas Red-conjugated goat anti-mouse IgM $\mu$ chain-specific secondary antibodies (Jackson ImmunoResearch). In some instances, tissue sections were counterstained with Thiazine Red (TR, $0.002 \%$ ) to visualize $\beta$-pleated sheet structures. All sections were then blocked with Sudan Black $(0.05 \%)$ to reduce autofluorescence. Images were captured using a Zeiss 510 laser scanning confocal microscope and all images were acquired as z-stacks of single optical sections.

\section{Results}

\section{Characterization of Nitration Site-Specific Novel Tau Antibodies}

To investigate whether nitration occurs at Y197 and Y394 in situ, we generated three novel monoclonal antibodies: Tau-nY197, Tau-Y197, and Tau-nY394. The specificity of these antibodies was determined using ELISA and Western blot analyses with wild-type tau (ht40), nitrated wildtype tau (nht40), and single tyrosine tau mutants nitrated at Y18, Y29, Y197, or Y394 (Figure 1). The Tau-nY197

\section{A}

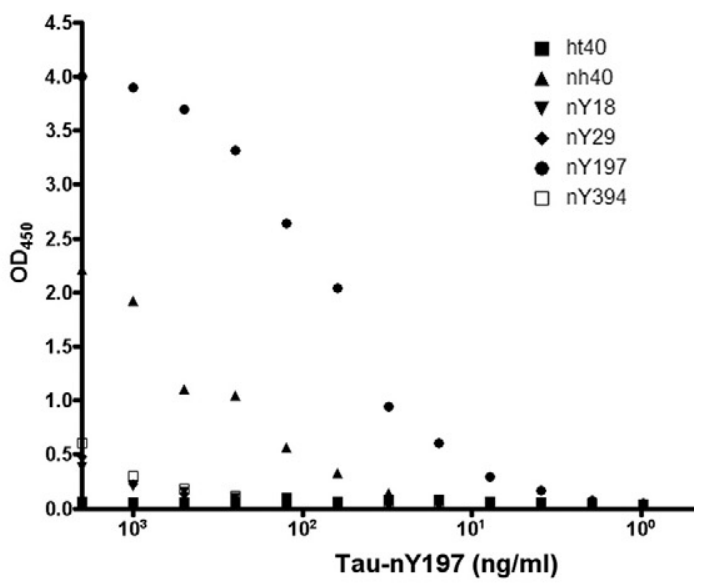

B
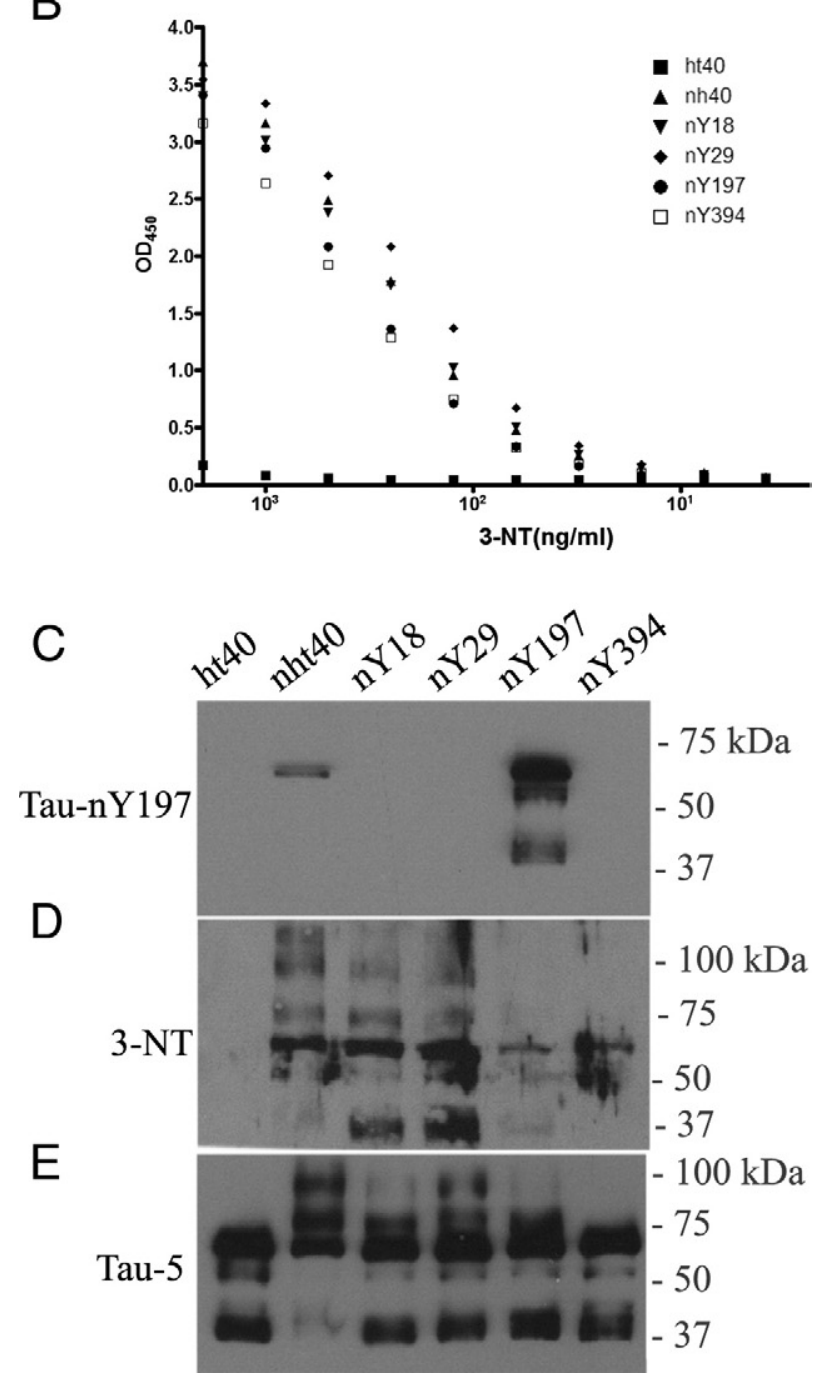

Figure 1. Nitration specificity of the Tau-nY197 antibody. The affinity of Tau-nY197 antibody against full-length human recombinant tau (ht40), fulllength nitrated tau (nht40), and tau proteins singly nitrated at Y18, Y29, Y197, and Y394 were determined by ELISA (A) and Western blot analysis (C). To confirm nitration within these samples, proteins were labeled with a generic nitrotyrosine antibody (3-NT) (B and D) and total tau was identified with Tau- $5(\mathbf{E})$, an antibody that labels tau proteins, regardless of the modification state. Note that Tau-nY197 reacts only with full-length nht 40 and nY197 tau. In all experiments, 50 and $100 \mathrm{ng}$ protein were used for Western blot and ELISA analyses, respectively. 
antibody (IgG1) recognized nitrated wild-type recombinant tau; among the single tyrosine mutants, it specifically recognized $\mathrm{nY} 197$ tau (Figure 1, A and C). To verify the addition of nitro groups to tyrosine residues after $\mathrm{ONOO}^{-}$treatment, a generic nitrotyrosine antibody was used (3-NT). The 3-NT antibody labeled nitrated wildtype tau and singly nitrated tyrosine proteins (Figure 1, B and D). Total tau within these samples was visualized using Tau- $5,{ }^{37}$ a pan tau antibody that recognizes tau, regardless of the modification state (Figure $1 \mathrm{E}$ ).

The specificity of Tau-nY197 was tested further by preabsorbing the antibody with either untreated or nitrated Y197 tau (nY197). As predicted, incubation with nY197 tau, but not with unmodified Y197 tau, abolished Tau-nY197 reactivity (see Supplemental Figure S1A at http://ajp.amjpathol.org). Last, to determine whether the nitro group comprised an essential portion of the TaunY197 epitope, the nitro moiety was reduced to an amine using dithionite (sodium hydrosulfite) treatment as described previously. ${ }^{31,35}$ Reduced proteins were no longer labeled with Tau-nY197 or 3-NT antibodies but were clearly reactive with Tau-5 (see Supplemental Figure S1B at $h$ ttp://ajp.amjpathol.org). These data indicate that the Tau-nY197 antibody selectivity is due, in part, to a nitrotyrosine group at position 197.

We also generated an antibody, Tau-Y197 (IgM), that specifically labels total tau but appears to require the hydroxyl group $(\mathrm{OH})$ of $\mathrm{Y} 197$ as part of its epitope (Figure 2). We suspect that this is the case because, in ELISA analysis, Tau-Y197 failed to react with tau proteins in which the tyrosine residue was mutated to phenylalanine $(\mathrm{Y} \rightarrow \mathrm{F})$, an amino acid lacking only the $\mathrm{OH}$ group in tyrosine residues (Figure 2A). Notably, both Tau-Y197 and Tau- 5 antibodies reacted with wild-type and single tyrosine recombinant tau proteins ( $Y 197)$, regardless of the nitration state (Figure 2, A and B). To further elucidate the specificity of this antibody in vitro, Y197 proteins were pseudo-phosphorylated by converting the tyrosine residue to glutamic acid $(\mathrm{Y} \rightarrow \mathrm{E})$ and tested for Tau-Y197 binding by ELISA. Tau-Y197 failed to recognize the pseudo-phosphorylated construct or Y197 constructs phosphorylated with the tyrosine kinase Abl (Figure 2, A and C). Collectively, these results demonstrate that the Tau-Y197 antibody labels nitrated or unmodified tau at Y197 but fails to label phosphorylated tau at the same position.

Finally, to demonstrate the specificity of Tau-nY394 (IgG2b) in vitro, ELISA and Western blot analyses were performed using nitrated tau proteins as above (Figure 3). As expected, Tau-nY394 detected nitrated wild-type tau and singly nitrated tau at Y394, but failed to react with untreated wild-type tau or with mutant proteins singly nitrated at Y18, Y29, or Y197 (Figure 3, A-C), thus demonstrating the site-selective specificity of Tau-nY394.

\section{Tau-nY197 and Tau-nY394 React with Nonprimate Tau}

Based on the high sequence homology of the proline-rich region in tau among human and other species (Figure 4A), we suspected that Tau-nY197 and Tau-nY394 would react
A

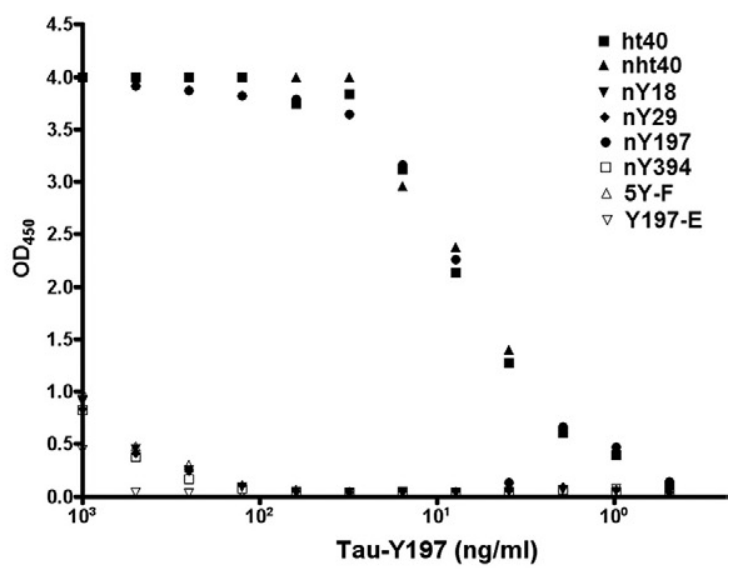

B

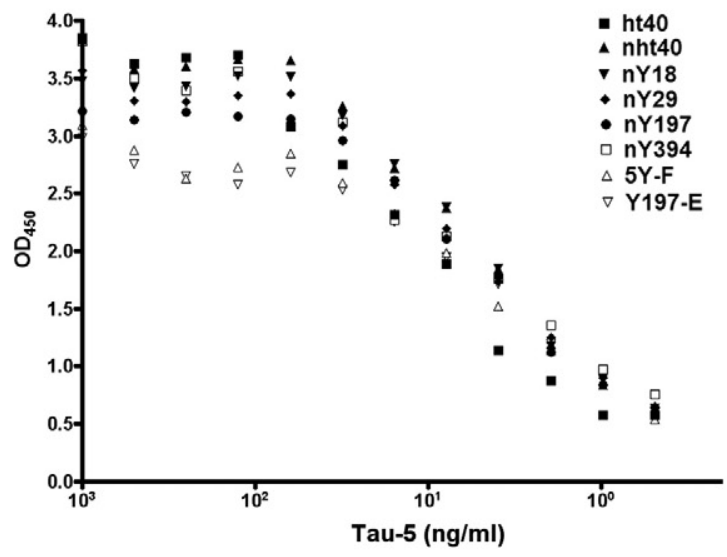

C

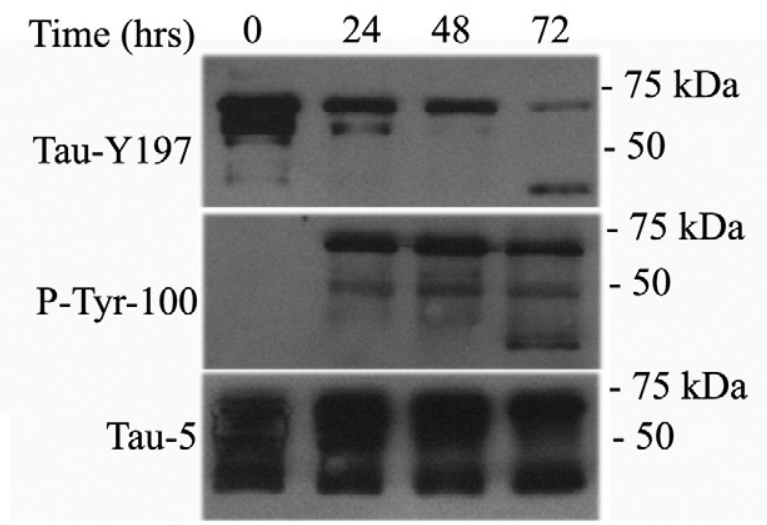

Figure 2. The Tau-Y197 antibody labels total tau, regardless of the nitration state. The binding of Tau-Y197 to full-length human recombinant tau (ht40), full-length nitrated tau (nht40), and tau proteins singly nitrated at Y18, Y29, Y197, and Y394 was assayed by ELISA. Note that Tau-Y197 labels all tau proteins containing Y197 regardless of the nitration state (A), but fails to detect $\mathrm{Y} \rightarrow \mathrm{E}$ pseudo-phosphorylated or tau proteins phosphorylated at Y197 by ELISA (A) or Western blot analysis (C). The addition of phospho-groups to proteins in vitro was confirmed using a generic phospho-tyrosine antibody (P-Tyr-100), and total tau within these samples was shown using Tau- 5 by ELISA (B) and Western blot techniques (C). Note that the OH group of Y197 appears to be an essential component of the Tau-Y197 epitope. In all experiments, 50 and 100 ng protein were used for Western blot and ELISA analyses, respectively.

with nonprimate tau after peroxynitrite treatment. To test this hypothesis, porcine microtubule fractions enriched for tau, MAP1A, MAP1B, and MAP2 were exposed to peroxynitrite and then blotted with 3-NT, Tau-nY197, or Tau-5 antibodies. 

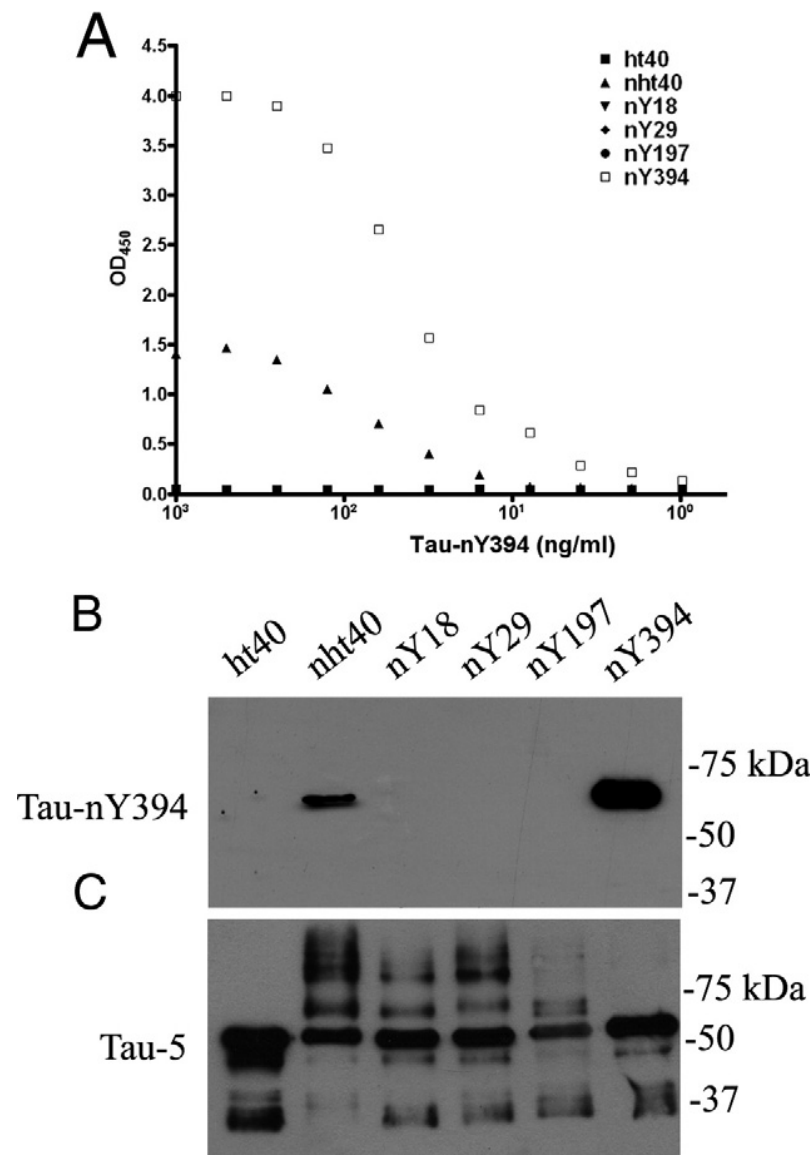

Figure 3. The Tau-nY394 antibody is specific for tau nitrated at Y394. Tau-nY394 reacts specifically with recombinant full-length nitrated wild-type proteins (nht40) and nitrated tau proteins singly nitrated at Y394 (nY394) by ELISA (A) and Western blot analysis (B), but fails to cross-react with other tau proteins singly nitrated at Y18, Y29, or Y197. Total tau was visualized by blotting with Tau-5 (C). In all experiments, 50 ng protein was used for Western blot and $100 \mathrm{ng}$ for ELISA analyses.

As expected, the 3-NT antibody recognized a smear of nitrated proteins after peroxynitrite treatment, thereby confirming the addition of nitro groups to tyrosine residues in all proteins present in vitro (Figure 4B). In contrast, Tau-nY197 only recognized porcine tau but only after peroxynitrite treatment, as confirmed by their cross-reactivity with Tau- 5 (Figure 4B). Similar results were obtained using bovine microtubule fractions and probing with both Tau-nY197 and Tau-nY394 antibodies (data not shown).

Next, we examined whether Tau-nY197 cross-reacts with proteins nitrated in other neurodegenerative diseases. Recombinant $\alpha$-synuclein and porcine tubulin ( $\alpha$ and $\beta$ ) were treated with peroxynitrite in vitro and then were blotted with $\alpha$-synuclein, $\beta$-tubulin, 3-NT, Tau-nY197, or Tau-nY394. As expected, both Tau-nY197 (Figure 4, C and D) and TaunY394 (data not shown) failed to cross-react with untreated or nitrated $\alpha$-synuclein and tubulin proteins.

\section{Tau Is Nitrated at Y197, Not at Y394, in Control and $A D$ Brain}

To determine whether nitration at Y197 or Y394 occurs in normal or $A D$ brain as it does in normal mouse brain, ${ }^{27}$ soluble tau from controls (Braak stages 0 to III) and severe $A D$ cases (Braak stages $V$ to $\mathrm{VI}$ ) (Table 1) was biochemically enriched, fractionated by SDS-polyacrylamide gel electrophoresis, and blotted with Tau-nY197 and TaunY394 antibodies. Western blot analyses revealed a subset of total tau migrating at approximately $50 \mathrm{kDa}$ that was nitrated at Y197 in both control and pathologically severe AD samples (Figure 5A). To exclude the possibility that phosphorylation within the Tau-nY197 epitope prevented antibody binding, because tau is highly susceptible to phosphorylation in this region in $A D,{ }^{38}$ nitrocellulose membranes containing soluble tau were treated with CIP before

A

Tau Proline Rich Region

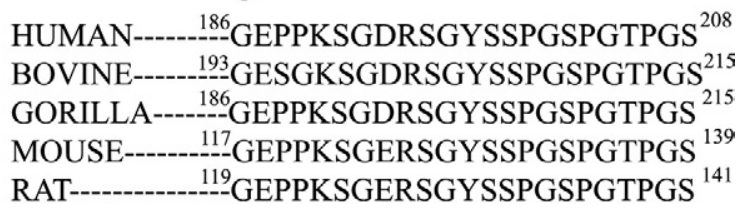

B

Porcine MAP fractions

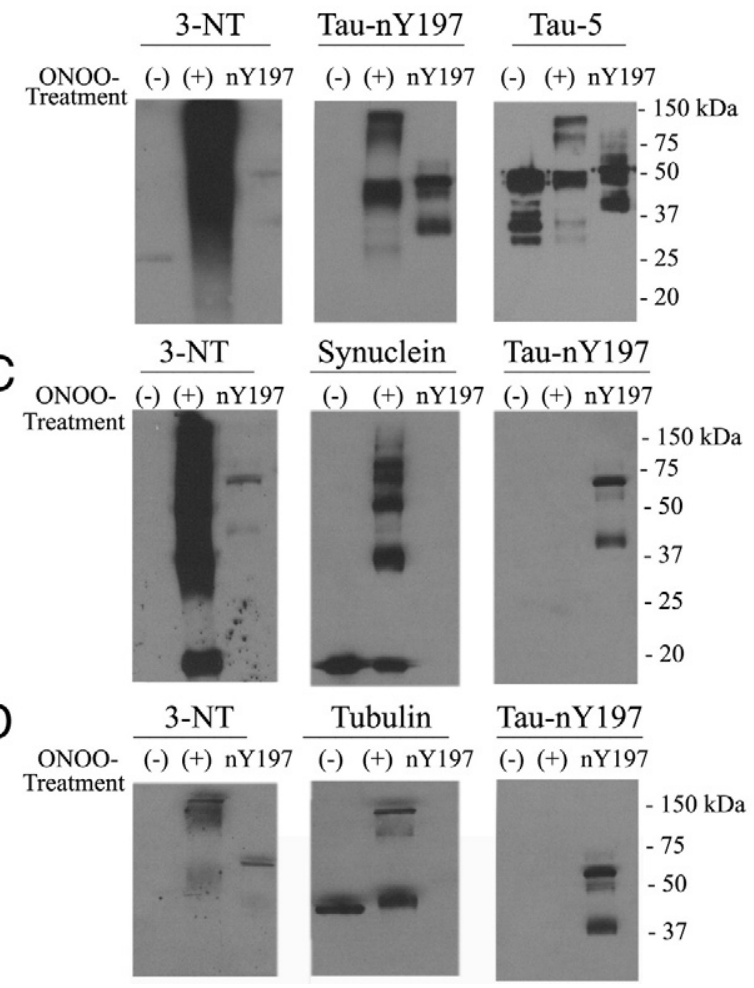

Figure 4. Tau-nY197 labels tau from nonprimate species. A: Tau protein sequence alignment shows the similarity in the proline-rich region of tau among different species. B: Porcine MAP fractions enriched for tau, MAP1A, MAP1B, and MAP2 were exposed to either vehicle $(-)$ or $\mathrm{ONOO}^{-}$treatment $(+)$, then were blotted with 3-NT, Tau-nY197, or Tau-5 antibodies. The 3-NT reactivity confirmed the nitration of all proteins by $\mathrm{ONOO}^{-}$. In contrast, Tau-nY97 failed to react with untreated porcine tau unless it was first exposed to peroxynitrite treatment $(+)$. Tau- 5 was used to confirm the presence of tau proteins in all samples. As expected, Tau-nY197 failed to crossreact with $\alpha$-synuclein $(\mathbf{C})$ or tubulin (D) proteins, regardless of the nitration state, but did label recombinant tau proteins selectively nitrated at Y197. For each porcine or singly nitrated recombinant sample, $1 \mu \mathrm{g}$ or $50 \mathrm{ng}$ was loaded per lane, respectively. 
A

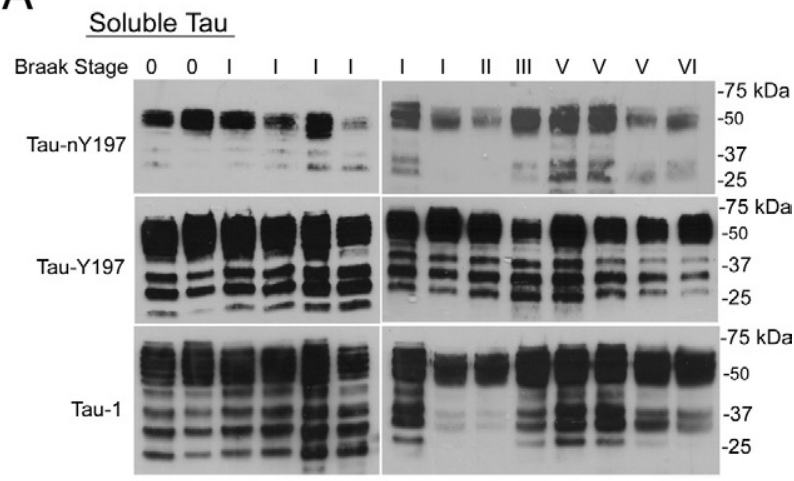

B

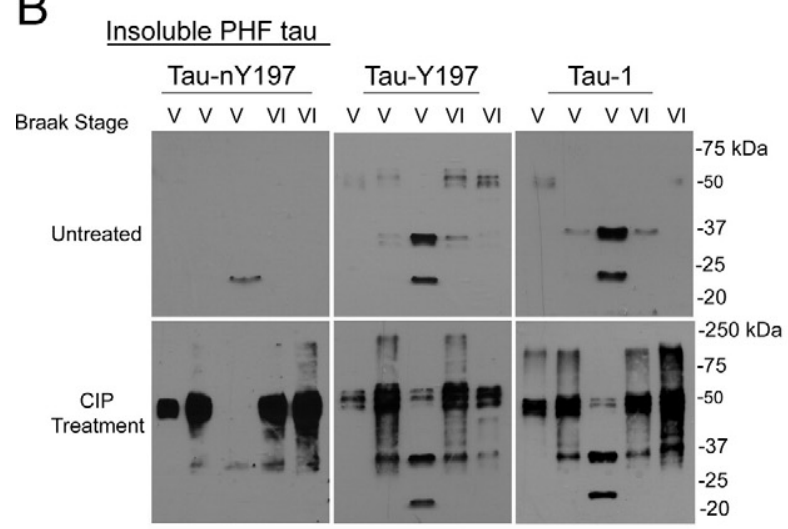

C

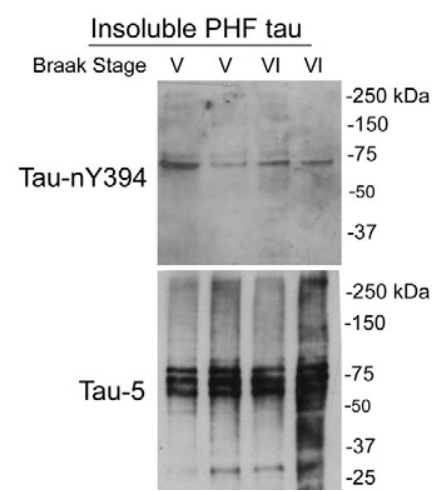

Figure 5. Tau nitration at tyrosine 197 occurs in normal human brain A: Western blot analysis of biochemically enriched soluble tau from controls (Braak stages 0 to III) and severe AD cases (Braak stages V to VI) showed a subset of total soluble tau nitrated at Y197, as indicated by Tau-nY197 reactivity. Total tau was labeled with Tau-Y197 and Tau-1. B: Insoluble PHF-tau fractions in samples from five patients with severe AD were probed with Tau-nY197, Tau-Y197, and Tau-1. Unlike the case for soluble tau, CIP treatment before antibody incubation was necessary to reveal the extent of insoluble PHF-tau nitrated at Y197 and total tau. C: Tau-nY394 labeled a small fraction of the PHF-tau, compared with the total tau labeled with Tau- 5 . In all samples, $10 \mu \mathrm{g}$ of total protein was loaded per lane.

antibody incubation. No differences were observed between treated or untreated membranes, indicating that soluble tau is likely not hyperphosphorylated in or around Y197 (data not shown). Total tau within these samples was labeled with Tau-Y197 and Tau-1, antibodies that have epitopes sensitive to phosphorylation and their epitopes overlap $^{33}$ (Figure 5A).
An identical analysis using our Tau-nY394 antibody was also performed on all soluble tau samples; however, this antibody failed to label any nitrated soluble tau from either control or severe AD samples, even when nitrocellulose membranes were pretreated with CIP prior to antibody incubation (see Supplemental Figure S2 at $h t t p: / /$ ajp.amjpathol.org). These data suggest that nitration at Y394 does not occur in soluble tau.

\section{Tau Is Nitrated at Both Y197 and Y394 in Insoluble PHF-Tau from AD Brain}

To determine whether insoluble tau fractions contained tau nitrated at Y197 or Y394, biochemically enriched PHF-tau from five severe AD samples (Table 1) was blotted with Tau-nY197 and Tau-nY394. Only a limited amount of nitrated tau was observed using Tau-nY197, Tau-Y197, and Tau-1 antibodies (Figure 5B). Unlike the case with soluble tau, CIP treatment before antibody incubation greatly increased the reactivity of all three antibodies for PHF-tau (Figure 5B). These data indicate that, within PHF-tau, phosphorylation events in or around the Tau-nY197 epitope prevent antibody binding much the same as they prevent binding of Tau-Y197 and Tau-1 antibodies. Finally, although Tau-nY394 failed to label soluble tau, trace amounts of insoluble PHF-tau were nY394-positive (Figure 5C); however, CIP treatment did not affect the reactivity of Tau-nY394 with PHF-tau (data not shown).

\section{Tau-nY197 Labels the Hallmark Tau Pathology of $A D$}

To investigate whether the pathological inclusions of $A D$ contained nitrated tau at residue Y197 or Y394, tissue sections from controls (Braak stages I to III) and severe $A D$ cases (Braak stages $V$ to $\mathrm{VI}$ ) were processed for $\mathrm{IHC}$ using Tau-nY197, Tau-nY394, or Tau-Y197 antibodies. Consistent with our Western blot results from PHF-tau, treatment of tissue sections with CIP before immunostaining increased Tau-nY197 reactivity. Thus, CIP treatment was used before antibody incubation in all samples analyzed. Immunohistochemical analysis in control sections (Braak stages I to III) revealed that neuropil threads, as well as a few NFTs, contained tau nitrated at Y197 (Figure $6, A, B, D$ and $E$ ). In later disease stages (Braak stages $V$ to $\mathrm{VI}$ ), the classic triad of tau inclusions (neuropil threads, neuritic plaques, and NFTs) were clearly positive for tau nitrated at Y197 (Figure 6, G and H). With increasing Braak stage, our qualitative observations revealed an increase in the Tau-nY197-positive tau inclusions, which were most prominent throughout the gray matter (Figure 6, B, E, and H). Importantly, Tau-nY197-positive inclusions were only a subset of the total tau lesions present within these sections, because extensive tau pathology in adjacent tissue sections was clearly labeled using TauY197, an antibody that does not discriminate between nitrated and non-nitrated tau (compare panel B to C, E to $F$, and $H$ to I in Figure 6). In all samples analyzed, however, Tau-nY394 failed to label the pathological lesions 


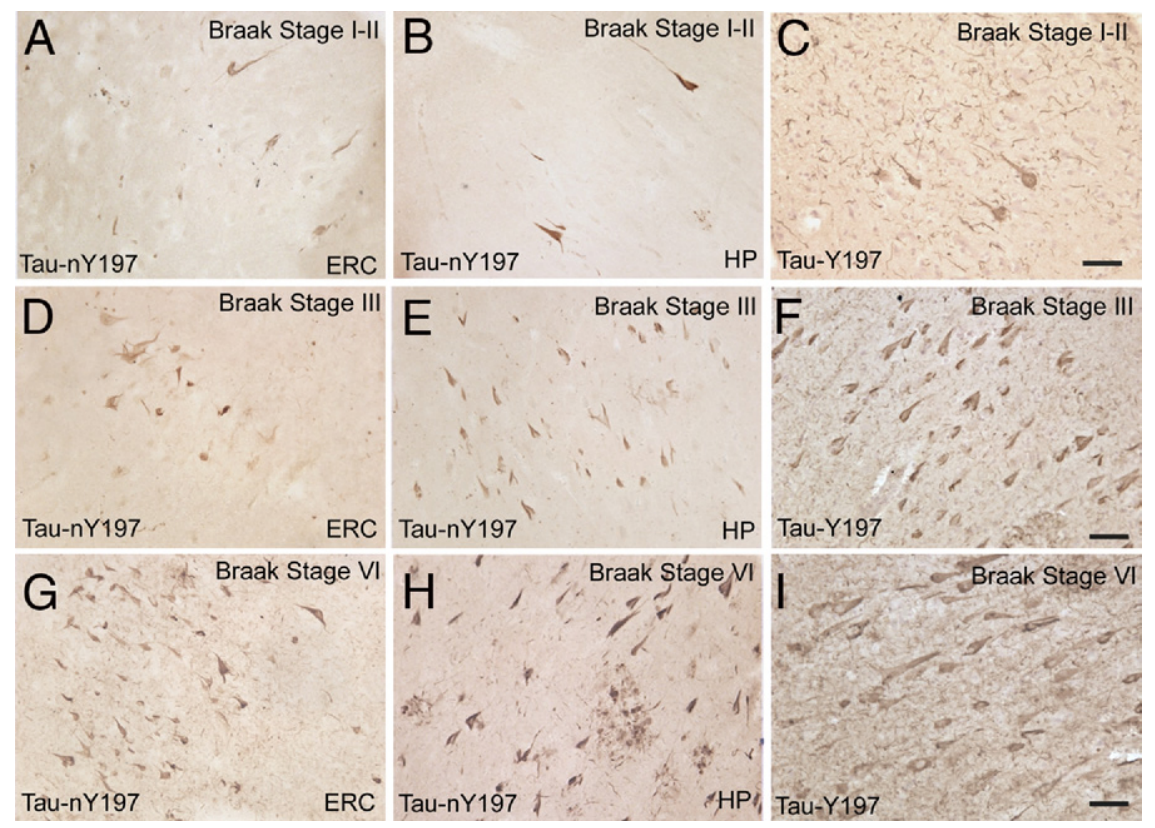

Figure 6. Tau-nY197 labels the hallmark tau pathology characteristic of AD. IHC was performed on CIP-treated tissue sections from the entorhinal cortex (ERC) and hippocampus (HP) of controls (Braak stages I to III) and severe AD cases (Braak stage VI) using Tau-nY197. In the ERC and HP from control sections, some neuropil threads as well as a few NFTs were labeled (A and $\mathbf{B}$ ) however, Tau-nY197 labeling increased considerably with disease progression $(\mathbf{D}, \mathbf{E}, \mathbf{G}$, and $\mathbf{H})$. Note that the Tau-nY197-positive inclusion. were only a subset of the total tau pathology, as revealed by Tau-Y197 staining in adjacent tissue sections ( $\mathbf{C}, \mathbf{F}$, and $\mathbf{I})$. Note also that neuropil threads, NFTs, and neuritic plaques stain with Tau-nY197 (H). Scale bar $=50 \mu \mathrm{m}$ (all panels)

characteristic of $A D$, even when CIP or several epitope retrieval methods (eg, formic or citric acid) were applied (data not shown). ${ }^{39}$ The lack of Tau-nY394 labeling in soluble tau and the relatively limited level of nitration at Y394 in PHF-tau suggest that Y394 nitration may be an infrequent modification in AD pathogenesis.

\section{Tau-nY197 Colocalizes with Both Early and Intermediate Markers of Tau Pathology}

To delineate whether the Tau-nY197-positive lesions associate with the early or late stages of tangle evolution in $A D$, double-label immunofluorescence was performed on CIP-treated sections using Tau-nY197 and Alz-50, a tau marker that labels pre-tangle neurons and early NFTs. ${ }^{40}$ In controls (Braak stage I to II), some Alz-50-positive neurons contained small granular tau inclusions that double-labeled with Tau-nY197, in what appeared to be the early stages of NFT formation (Figure 7, A-C). However, extensive colocalization was observed in more mature fibrillar NFTs of Braak stage III brain (Figure 7, D-F). In severe AD cases (Braak stage VI), Tau-nY197-positive inclusions were sparsely labeled with Alz-50 (data not shown), but showed substantial overlap with Tau-C3 (Figure 7, G-I), a tau marker that temporally follows the Alz-50 conformational shift in the evolution of NFTs in AD.9,40 Additionally, TaunY197 colocalized with the pS422 tau antibody, a pathological tau phospho-epitope that is thought to occur in pretangle neurons (data not shown). ${ }^{40,41}$

To further investigate whether the nitrated tau lesions labeled with Tau-nY197 associate with $\beta$-pleated sheet structures, tissue sections were stained with Tau-nY197 and TR. Of note, within the limited number of TR-positive inclusions observed in control sections (Braak stage I to II), some contained small granular aggregates that were positive for Tau-nY197 (Figure 7, J-L). In severe AD cases (Braak stage VI), Tau-nY197-positive lesions also colocalized with TR, but not all Tau-nY197-positive inclusions were TR-positive (Figure 7, M-O). Importantly, in all $A D$ sections analyzed, the pathological lesions labeled with Tau-nY197 colocalized partially with Tau-Y197 (Figure 8, A-C), confirming that the inclusions labeled with Tau-nY197 were composed at least in part of nitrated tau. Notably, similar to Tau-nY197, Tau-Y197 also colocalized extensively with Tau-C3 in control (Figure 8, D-F) and $A D$ samples alike (Figure 8, G-I). However, the epitope display for Tau-Y197 appears to emerge much earlier than that of Tau-C3 in AD, as shown by the diffusely labeled hippocampal neurons (Figure 8D) and early labeling of numerous neuropil threads (Figure 6C). Notably, both Tau-nY197 and Tau-Y197 did not colocalize with the MN423 antibody (Figure 8, J-L), a tau marker of the latest known stages of tangle evolution. ${ }^{40}$ Hence, our data indicate that nitration at Y197 can occur coincident with tau markers of the early (Alz-50) and intermediate stages (Tau-C3) of AD pathology but disappears by the end stages (MN423) of tangle evolution.

\section{Discussion}

\section{Timing of Nitration during Tangle Evolution in $A D$ Pathogenesis}

Here we have reported the characterization of two nitration site-specific monoclonal antibodies that selectively identify tau nitrated at either Y197 (Tau-nY197) or Y394 (Tau-nY394). Both antibodies were stringently tested to confirm their specificity for single nitrated tyrosine sites within tau, and we ruled out potential cross-reactivity with other proteins (eg, $\alpha$-synuclein and tubulin) nitrated in other neurodegenerative diseases. Notably, Tau-nY197 identified nitrated soluble tau in every sample assayed, with classifications ranging from Braak stage 0 through Braak stage VI. 


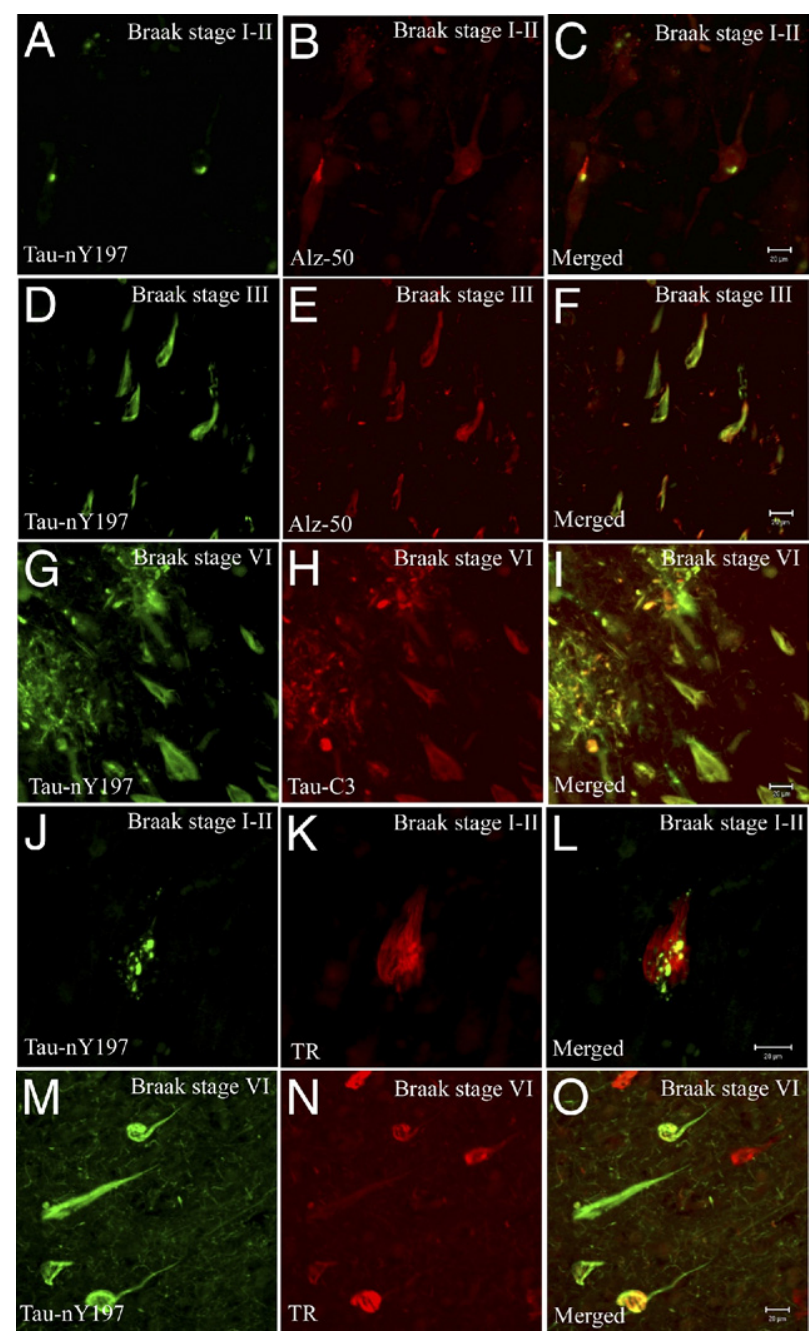

Figure 7. Tau-nY197 colocalizes with tau markers of the initial stages of tangle formation in AD. Double-label immunofluorescence was performed on CIP-treated tissue sections in areas of the HP from control samples (Braak stages I to II) using Tau-nY197 and Alz-50 antibodies. Tau-nY197 labeled small tau aggregates within Alz-50-positive neurons (A-C), as well as the fibrillar lesions in Braak stage III cases (which also were Alz-50-positive) (D-F). Tau-nY197 also colocalized with Tau-C3 within the fibrillar lesions in severe (Braak stage VI) cases (G-I). Thiazine Red (TR) staining of control sections (Braak stages I to II) showed small tau aggregates labeled by Tau-nY197 that colocalized within TR-positive NFTs (J-L). In severe cases (Braak stage VI), some but not all of the Tau-nY197-positive lesions were TR-positive (M-O). Scale bar $=20 \mu \mathrm{m}$ (all panels)

Tau-nY197 labeled nitrated tau within the hallmark fibrillar lesions found in normal controls and severe disease states alike. In fact, extensive colocalization was observed with monoclonal antibodies that recognize modified tau during the initial and middle stages of NFT formation (Alz-50, pS422, and Tau-C3). ${ }^{40}$ This was not surprising, given that Y197-nitrated tau was present in the soluble state independent of disease or pathological designation. Of note, TaunY197 did not colocalize with MN423, a proteolytic cleavage event marking the latest known stage of tangle evolution in AD. ${ }^{40}$ The lack of colocalization between TaunY197 or Tau-Y197 with the MN423 antibody might be due to a proteolytic cleavage that ablates position 197, perhaps generated by a chymotrypsin-like endopeptidase. ${ }^{42,43} \mathrm{Col}$ lectively, these data indicate that nitration at Y197 occurs before the appearance of detectable tau pathology and colocalizes with only a subset of tau pathology during the initial stages of tangle formation in AD.

The lack of nitration at Y394 in soluble tau and the limited nitration in insoluble PHF-tau suggest that nitration at Y394 is likely an infrequent event in AD. Nonetheless, it is certainly possible that proteolytic cleavage at amino acid 391 in AD recognized by the MN423 antibody ${ }^{44}$ might eliminate TaunY394 reactivity, should it occur late in NFT evolution. Alternatively, phosphorylation at Y394 has previously been reported in $A D,{ }^{45}$ and this may explain the limited nitration at this site. The highly electronegative orthophosphate group likely would not permit the addition of a nitro group at the meta position of the same tyrosine ring. A balance between nitration and phosphorylation at specific amino acids may have some biological importance, ${ }^{26,46}$ but this hypothesis requires further study.

\section{Is Nitration at Tyrosine 197 a Normal or Pathological Event in the Human Brain?}

Tyrosine nitration is a posttranslational modification widely thought of as a pathological event associated with

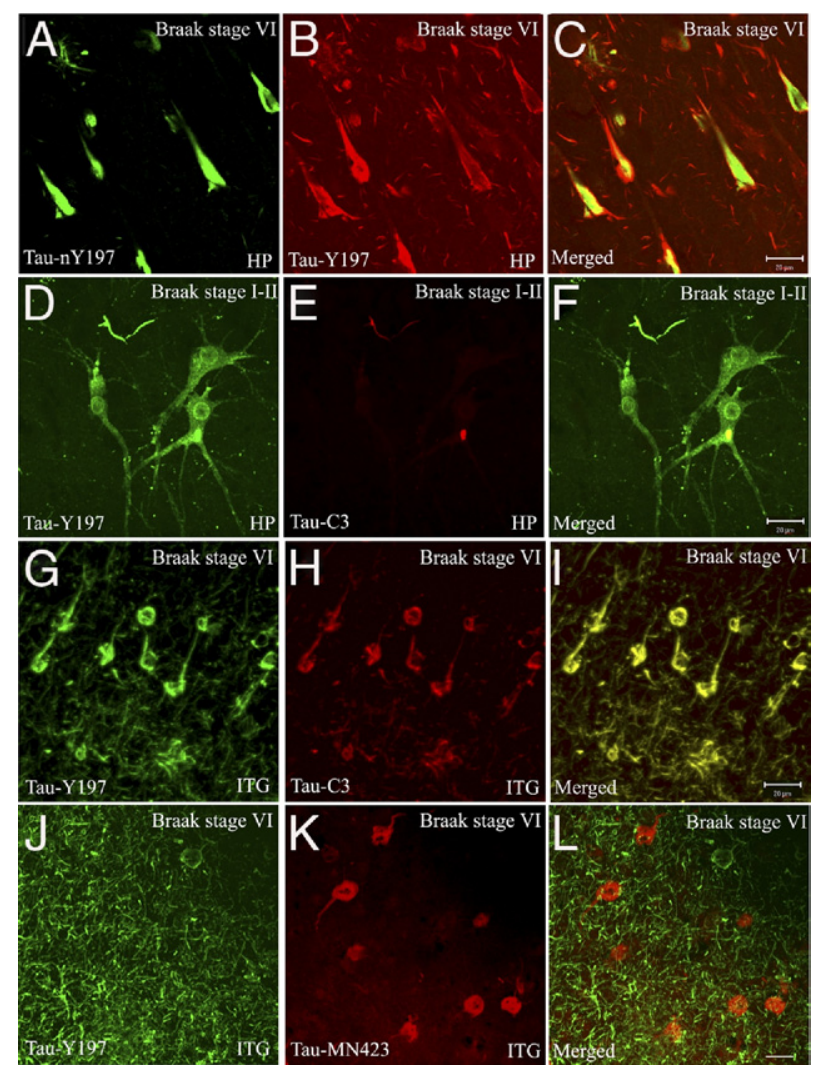

Figure 8. Tau-Y197 colocalizes partially with Tau-nY197, but fails to colocalize with a late tau marker of tangle evolution in AD. CIP-treated tissue sections from areas of the HP and ITG from severe AD samples (Braak stage VI) were labeled with Tau-Y197 and Tau-nY197. In all samples analyzed, Tau-Y197 colocalized partially with Tau-nY197 (A-C). However, extensive colocalization was observed with Tau-C3-positive aggregates that were present within neurons labeled with Tau-Y197 in controls (D-F). In severe AD (Braak stage VI), the fibrillar tau lesions labeled with Tau-Y197 also colocalized extensively with Tau-C3 (G-I). However, Tau-Y197 failed to colocalize in most instances with MN423 (J-L). Scale bars $=20 \mu \mathrm{m}$ (all panels). 
inflammation and pathogenesis in various neurodegenerative disorders. ${ }^{12,14}$ On the other hand, recent findings support a normal biological function for tyrosine nitration within cells. ${ }^{25-28}$ Tyrosine nitration has been suggested to play a role in neurite elongation and differentiation in neuronal cell types. ${ }^{47}$ Most recently, nitration of tau at Y197 has been reported in brain from 6-week-old wildtype mice, based on mass-spectrometry analysis. ${ }^{27}$ Of note, Rayala et $\mathrm{al}^{46}$ identified a dynamic interplay between nitration and phosphorylation in the control of microtubule dynamics in situ, suggesting that tyrosine nitration might have some normal biological function.

In addition to nitration at Y197 (the present study), Y197 phosphorylation has been identified in AD and in a mouse model of tauopathy (for a review, see Lebouvier et $\mathrm{al}^{48}$ ), indicating that both events occur within the context of $A D$. Although the explanation for these findings remains obscure, it is possible that different isoforms of tau are more or less susceptible to phosphorylation and/or nitration during the disease process. Moreover, the identification of tau nitrated at Y197 in brain from normal control subjects suggests that certain nitration events may be nonpathological. Similar to phosphorylation, tyrosine nitration may be a normal modification that increases in frequency in $A D$ or is concentrated within $A D$ pathology. In fact, several tau phospho-epitopes are known to occur in both fetal and normal aging brain, but these epitopes appear to be stabilized in disease-associated tau aggregates (for a review, see Liu et $\mathrm{al}^{49}$ ). However, other sites appear to be specifically phosphorylated only in AD. ${ }^{50}$ Similarly, nitration at $Y 197$ and $Y 18$ in tau, which was observed in normal age-matched control subjects, appears to increase with disease progression as the nitrated monomers are incorporated into tau aggregates. ${ }^{32}$ By contrast, nitration of tau at $Y 29$ was observed only in $A D,{ }^{31}$ much like the disease-specific phosphorylation sites. ${ }^{50}$ These studies collectively suggest that tyrosine nitration behaves in a manner similar to phosphorylation, in that some tyrosine sites are susceptible to nitration only in $A D$ and nitration at other sites occurs in both normal control subjects and AD patients. Unlike phosphorylation, the underlying mechanism and/or the normal biological function for tyrosine nitration remain unknown.

\section{Acknowledgments}

We thank Bryan Hitt, B.A., from the laboratory of Dr. Robert Vassar and the members of the Binder Laboratory (Feinberg School of Medicine, Northwestern University, Chicago, IL) for critical review of this manuscript. We also thank Dr. Eileen Bigio and Dr. Changiz Geula (the Cognitive Neurology and Alzheimer's Disease Center, Feinberg School of Medicine, Northwestern University), Dr. Anne McKee (Boston University School of Medicine and Alzheimer's Disease Center, Boston, MA), and Dr. Elliot Mufson from the Department of Neurological Sciences (Rush University, Chicago, IL), for kindly providing human brain tissue.

\section{References}

1. Glenner GG, Wong CW: Alzheimer's disease: initial report of the purification and characterization of a novel cerebrovascular amyloid protein. Biochem Biophys Res Commun 1984, 120:885-890

2. Grundke-Iqbal I, Iqbal K, Quinlan M, Tung YC, Zaidi MS, Wisniewski HM: Microtubule-associated protein tau. A component of Alzheimer paired helical filaments. J Biol Chem 1986, 261:6084-6089

3. Goedert M, Wischik CM, Crowther RA, Walker JE, Klug A: Cloning and sequencing of the cDNA encoding a core protein of the paired helical filament of Alzheimer disease: identification as the microtubule-associated protein tau. Proc Natl Acad Sci USA 1988, 85:40514055

4. Goedert M, Spillantini MG, Jakes R, Rutherford D, Crowther RA: Multiple isoforms of human microtubule-associated protein tau: sequences and localization in neurofibrillary tangles of Alzheimer's disease. Neuron 1989, 3:519-526

5. Ksiezak-Reding H, Liu WK, Yen SH: Phosphate analysis and dephosphorylation of modified tau associated with paired helical filaments. Brain Res 1992, 597:209-219

6. Braak H, Braak E: Neuropathological stageing of Alzheimer-related changes. Acta Neuropathol 1991, 82:239-259

7. Arriagada PV, Growdon JH, Hedley-Whyte ET, Hyman BT: Neurofibrillary tangles but not senile plaques parallel duration and severity of Alzheimer's disease. Neurology 1992, 42:631-639

8. Abraha A, Ghoshal N, Gamblin TC, Cryns V, Berry RW, Kuret J, Binder LI: C-terminal inhibition of tau assembly in vitro and in Alzheimer's disease. J Cell Sci 2000, 113:3737-3745

9. Gamblin TC, Chen F, Zambrano A, Abraha A, Lagalwar S, Guillozet AL, Lu M, Fu Y, Garcia-Sierra F, LaPointe N, Miller R, Berry RW, Binder LI, Cryns VL: Caspase cleavage of tau: linking amyloid and neurofibrillary tangles in Alzheimer's disease. Proc Natl Acad Sci USA 2003, 100:10032-10037

10. Park SY, Ferreira A: The generation of a $17 \mathrm{kDa}$ neurotoxic fragment: an alternative mechanism by which tau mediates beta-amyloid-induced neurodegeneration. J Neurosci 2005, 25:5365-5375

11. Carmel G, Mager EM, Binder LI, Kuret J: The structural basis of monoclonal antibody Alz50's selectivity for Alzheimer's disease pathology. J Biol Chem 1996, 271:32789-32795

12. Horiguchi T, Uryu K, Giasson BI, Ischiropoulos $H$, LightFoot R, Bellmann C, Richter-Landsberg C, Lee VM, Trojanowski JQ: Nitration of tau protein is linked to neurodegeneration in tauopathies [Erratum appeared in Am J Pathol 2003, 163:2645]. Am J Pathol 2003, 163: 1021-1031

13. Reynolds MR, Berry RW, Binder LI: Nitration in neurodegeneration: deciphering the "Hows" "nYs". Biochemistry 2007, 46:7325-7336

14. Beckman JS, Chen J, Crow JP, Ye YZ: Reactions of nitric oxide, superoxide and peroxynitrite with superoxide dismutase in neurodegeneration. Prog Brain Res 1994, 103:371-380

15. Smith MA, Richey Harris PL, Sayre LM, Beckman JS, Perry G: Widespread peroxynitrite-mediated damage in Alzheimer's disease. J Neurosci 1997, 17:2653-2657

16. Beckman JS, Koppenol WH: Nitric oxide, superoxide, and peroxynitrite: the good, the bad, and ugly. Am J Physiol 1996, 271: C1424-C1437

17. Thomas DD, Miranda KM, Colton CA, Citrin D, Espey MG, Wink DA: Heme proteins and nitric oxide (NO): the neglected, eloquent chemistry in NO redox signaling and regulation. Antioxid Redox Signal 2003, 5:307-317

18. Pfeiffer S, Lass A, Schmidt K, Mayer B: Protein tyrosine nitration in mouse peritoneal macrophages activated in vitro and in vivo: evidence against an essential role of peroxynitrite. FASEB J 2001, 15: 2355-2364

19. Thomas DD, Ridnour LA, Isenberg JS, Flores-Santana W, Switzer $\mathrm{CH}$, Donzelli S, Hussain P, Vecoli C, Paolocci N, Ambs S, Colton CA, Harris CC, Roberts DD, Wink DA: The chemical biology of nitric oxide: implications in cellular signaling. Free Radic Biol Med 2008, 45:18-31

20. Ischiropoulos $\mathrm{H}$, al-Mehdi AB: Peroxynitrite-mediated oxidative protein modifications. FEBS Lett 1995, 364:279-282

21. Beckman JS, Carson M, Smith CD, Koppenol WH: ALS, SOD and peroxynitrite. Nature 1993, 364:584

22. Atwood CS, Perry G, Zeng $H$, Kato $Y$, Jones WD, Ling KQ, Huang $X$, Moir RD, Wang D, Sayre LM, Smith MA, Chen SG, Bush AI: Copper 
mediates dityrosine cross-linking of Alzheimer's amyloid-beta. Biochemistry 2004, 43:560-568

23. Souza JM, Giasson BI, Chen Q, Lee VM, Ischiropoulos H: Dityrosine cross-linking promotes formation of stable alpha-synuclein polymers. Implication of nitrative and oxidative stress in the pathogenesis of neurodegenerative synucleinopathies. J Biol Chem 2000, 275: $18344-18349$

24. Reynolds MR, Berry RW, Binder LI: Site-specific nitration and oxidative dityrosine bridging of the tau protein by peroxynitrite: implications for Alzheimer's disease. Biochemistry 2005, 44:1690-1700

25. Aulak KS, Koeck T, Crabb JW, Stuehr DJ: Dynamics of protein nitration in cells and mitochondria. Am J Physiol Heart Circ Physiol 2004, 286: $\mathrm{H} 30-\mathrm{H} 38$

26. Gow AJ, Farkouh CR, Munson DA, Posencheg MA, Ischiropoulos H: Biological significance of nitric oxide-mediated protein modifications Am J Physiol Lung Cell Mol Physiol 2004, 287:L262-L268

27. Nonnis S, Cappelletti G, Taverna F, Ronchi C, Ronchi S, Negri A, Grassi E, Tedeschi G: Tau is endogenously nitrated in mouse brain: identification of a tyrosine residue modified in vivo by NO. Neurochem Res 2008, 33:518-525

28. Giannopoulou E, Katsoris P, Polytarchou C, Papadimitriou E: Nitration of cytoskeletal proteins in the chicken embryo chorioallantoic membrane. Arch Biochem Biophys 2002, 400:188-198

29. Kamisaki Y, Wada K, Bian K, Balabanli B, Davis K, Martin E, Behbod F, Lee YC, Murad F: An activity in rat tissues that modifies nitrotyrosine-containing proteins. Proc Natl Acad Sci USA 1998, 95:1158411589

30. Reynolds MR, Berry RW, Binder LI: Site-specific nitration differentially influences tau assembly in vitro. Biochemistry 2005, 44:13997-14009

31. Reynolds MR, Reyes JF, Fu Y, Bigio EH, Guillozet-Bongaarts AL, Berry RW, Binder LI: Tau nitration occurs at tyrosine 29 in the fibrillar lesions of Alzheimer's disease and other tauopathies. J Neurosci 2006, 26:10636-10645

32. Reyes JF, Reynolds MR, Horowitz PM, Fu Y, Guillozet-Bongaarts AL, Berry R, Binder LI: A possible link between astrocyte activation and tau nitration in Alzheimer's disease. Neurobiol Dis 2008, 31:198-208

33. Binder LI, Frankfurter A, Rebhun LI: The distribution of tau in the mammalian central nervous system. J Cell Biol 1985, 101:1371-1378

34. Hanger DP, Betts JC, Loviny TL, Blackstock WP, Anderton BH: New phosphorylation sites identified in hyperphosphorylated tau (paired helical filament-tau) from Alzheimer's disease brain using nanoelectrospray mass spectrometry. J Neurochem 1998, 71:2465-2476

35. Ye YZ, Strong M, Huang ZQ, Beckman JS: Antibodies that recognize nitrotyrosine. Methods Enzymol 1996, 269:201-209

36. Mufson EJ, Lavine N, Jaffar S, Kordower JH, Quirion R, Saragovi HU: Reduction in p140-TrkA receptor protein within the nucleus basalis and cortex in Alzheimer's disease. Exp Neurol 1997, 146:91-103

37. LoPresti P, Szuchet S, Papasozomenos SC, Zinkowski RP, Binder LI: Functional implications for the microtubule-associated protein tau: localization in oligodendrocytes. Proc Natl Acad Sci USA 1995, 92 : 10369-10373
38. Eidenmüller J, Fath T, Maas T, Pool M, Sontag E, Brandt R: Phosphorylation-mimicking glutamate clusters in the proline-rich region are sufficient to simulate the functional deficiencies of hyperphosphorylated tau protein. Biochem J 2001, 357:759-767

39. Pellicer EM, Sundblad A: Recuperación de antígenos por horno de microondas con buffer de ácido cítrico [Antigen retrieval by microwave oven with buffer of citric acid]. Spanish. Medicina (B Aires) 1994, 54:129-132

40. Guillozet-Bongaarts AL, Garcia-Sierra F, Reynolds MR, Horowitz PM, Fu Y, Wang T, Cahill ME, Bigio EH, Berry RW, Binder LI: Tau truncation during neurofibrillary tangle evolution in Alzheimer's disease. Neurobiol Aging 2005, 26:1015-1022

41. Bussière $T$, Hof PR, Mailliot C, Brown CD, Caillet-Boudin ML, Perl DP, Buée L, Delacourte A: Phosphorylated serine422 on tau proteins is a pathological epitope found in several diseases with neurofibrillary degeneration. Acta Neuropathol 1999, 97:221-230

42. Steiner B, Mandelkow EM, Biernat J, Gustke N, Meyer HE, Schmidt B, Mieskes G, Söling HD, Drechsel D, Kirschner MW, Goedert M, Mandelkow E: Phosphorylation of microtubule-associated protein tau: identification of the site for $\mathrm{Ca} 2(+)$-calmodulin dependent kinase and relationship with tau phosphorylation in Alzheimer tangles. EMBO $J$ 1990, 9:3539-3544

43. Wang Y, Garg S, Mandelkow EM, Mandelkow E: Proteolytic processing of tau. Biochem Soc Trans, 2010, 38:955-961

44. Khuebachova M, Verzillo V, Skrabana R, Ovecka M, Vaccaro P, Panni S, Bradbury A, Novak M: Mapping the C terminal epitope of the Alzheimer's disease specific antibody MN423. J Immunol Methods 2002, 262:205-215

45. Tremblay MA, Acker CM, Davies P: Tau phosphorylated at tyrosine 394 is found in Alzheimer's disease tangles and can be a product of the Abl-related kinase, Arg. J Alzheimers Dis 2010, 19:721-733

46. Rayala SK, Martin E, Sharina IG, Molli PR, Wang X, Jacobson R, Murad F, Kumar R: Dynamic interplay between nitration and phosphorylation of tubulin cofactor B in the control of microtubule dynamics [Erratum appeared in Proc Natl Acad Sci USA 2008, 105:399]. Proc Natl Acad Sci USA 2007, 104:19470-19475

47. Cappelletti G, Maggioni MG, Ronchi C, Maci R, Tedeschi G: Protein tyrosine nitration is associated with cold- and drug-resistant microtubules in neuronal-like PC12 cells. Neurosci Lett 2006, 401:159-164

48. Lebouvier T, Scales TM, Williamson R, Noble W, Duyckaerts C, Hanger DP, Reynolds CH, Anderton BH, Derkinderen P: The microtubule-associated protein tau is also phosphorylated on tyrosine. $J$ Alzheimers Dis 2009, 18:1-9

49. Liu F, Liang Z, Gong CX: Hyperphosphorylation of tau and protein phosphatases in Alzheimer disease. Panminerva Med 2006, 48: 97-108

50. Zheng-Fischhöfer Q, Biernat J, Mandelkow EM, Illenberger S, Godemann R, Mandelkow E: Sequential phosphorylation of Tau by glycogen synthase kinase-3beta and protein kinase A at Thr212 and Ser214 generates the Alzheimer-specific epitope of antibody AT100 and requires a paired-helical-filament-like conformation. Eur J Biochem 1998, 252:542-552 